\title{
LOCALLY CONSTANT COHOMOLOGY
}

\author{
E. SPANIER
}

\begin{abstract}
In this paper we study locally constant cohomology theories on a space $X$. We prove that for cohomology theories on a category of paracompact spaces the homotopy axiom of Eilenberg-Steenrod is a consequence of the other Eilenberg-Steenrod axioms together with continuity and either additivity or weak additivity. We also prove that if $H$ is a cohomology theory on the space of a simplicial complex $K$ which is locally constant on every open simplex of $K$ there is a spectral sequence converging to $H(|K|)$ whose $E_{2}$-term is the usual simplicial cohomology of $K$ with coefficients in various stacks on $K$ defined by $H$. This generalizes some known spectral sequences.
\end{abstract}

\section{INTRODUCTION}

Let $\operatorname{cl}(X)$ denote the category of all closed subsets of the topological space $X$ (in this paper all topological spaces will be Hausdorff spaces) and inclusion maps between them. A cohomology theory $H, \delta$ on $X[5,6,7,8]$ consists of a contravariant functor $H$ from $\operatorname{cl}(X)$ to the category of graded abelian groups together with a natural transformation $\delta: H(A \cap B) \rightarrow H(A \cup B)$ of degree 1 for $A, B \in \operatorname{cl}(X)$ satisfying some simple axioms. For such cohomology theories there are comparison theorems having wide applicability in topology $[5,8]$.

A natural question asks for all cohomology theories in the above sense on a space $X$. This does not appear feasible at the present time. In this paper we attempt to analyze those which are locally constant (roughly speaking, a cohomology theory is locally constant if every point has a closed neighborhood such that the cohomologies of every two points in the neighborhood are isomorphic). Every contravariant functor $H$ on $\operatorname{cl}(X)$ which is the restriction to $\operatorname{cl}(X)$ of a functor from the category of closed subsets of $X$ and all continuous maps between them is locally constant on $X$ (see (2.4) below).

Thus, there are locally constant cohomology theories and results about them are valid for the usual cohomology theories defined on a category of topological spaces and continuous maps (satisfying the Eilenberg-Steenrod axioms other than the dimension axiom). As one particular consequence of our study of locally constant cohomology theories we deduce homotopy invariance on the

Received by the editors November 15, 1989.

1980 Mathematics Subject Classification (1985 Revision). Primary 55N40; Secondary 55N20, $55 \mathrm{~N} 30,55 \mathrm{~T} 25$.

The author gratefully acknowledges partial support by the Centre de Recerca Mathematica of the Institut d'Estudis Catalans (Barcelona), Spain during the preparation of this paper. 
category of paracompact spaces and continuous maps between them for cohomology theories which are nonnegative, continuous, and additive or weakly additive [8]. This is known [2] for continuous cohomology theories on the category of compact spaces (on a compact space every cohomology theory is additive and weakly additive). The proof of the result in the compact case does not use other properties of cohomology. In fact, every continuous contravariant functor from the category of compact spaces to the category of groups is invariant under homotopy. However, our proof of homotopy invariance in the paracompact space uses in an essential way the other properties of cohomology inasmuch as it entails use of comparison theorems.

Finally, we specialize to cohomology theories on simplicial complexes which are locally constant on every open simplex. We prove a limit property relating $H(|K|)$ to limits of $H\left(\left|K^{p}\right|\right)$ as $p$ varies (see (5.8) below) and use this to construct a spectral sequence converging to $H(|K|)$ whose $E_{2}$-term is isomorphic to the cohomology of $K$ defined by $H$ (see (6.9) below). When applied to a usual cohomology theory (such as $K$-theory on a finite dimensional polyhedron) the spectral sequence generalizes the Atiyah-Hirzebruch spectral sequence for a finite complex. Another application is to $f: X \rightarrow Y$ where $f$ is a closed simplicial map or a closed projection of a filtration whose base is a polyhedron. In this case there is a cohomology theory $f_{*} H$ on $Y$ defined by $\left(f_{*} H\right)(B)=H\left(f^{-1}(B)\right)$ (Čech cohomology for some fixed coefficient group) (see (5.1) or (5.2) below). In the first instance the spectral sequence is a form of Leray spectral sequence for the map $f$, and, in the second instance, it is a form of Serre spectral sequence for the projection $p$. In both of these cases, if $Y$ is finite dimensional, the same considerations apply to any cohomology theory $H$ on $X$ which is invariant under homotopy (for example, to $K$-theory). In this more general case the spectral sequence converges to $H(X)$ and its $E_{2}$ term is isomorphic to the simplicial cohomology of $Y$ with coefficients in stacks defined by $f$ and $H$. This spectral sequence appears to be new.

If $X$ is a polyhedron there is a cohomology theory

$$
{ }^{\Delta} H^{q}(A)=H_{-q}(X, X-A)
$$

(singular homology with some fixed coefficient group or local system of groups) (see (5.3) below). In case $X$ is finite dimensional the corresponding spectral sequence converges to $H_{*}(X)$ and its $E_{2}$-term is isomorphic to the cohomology of $X$ with coefficients in stacks defined in terms of homology. This again seems to be new.

In this paper we do not pursue these special spectral sequences but, as one application, we prove a theorem generalizing the uniqueness theorem of Milnor [3] for additive cohomology theories on simplicial complexes (see (6.10) below).

The rest of the paper is divided into five sections. Section 2 is devoted to contravariant functors on $\operatorname{cl}(X)$ and contains the definition of local constancy for these functors and discussion of some of their elementary properties. In $\S 3$ locally constant cohomology theories are considered, and a proof is given for the homotopy invariance (Theorem (3.4) ) referred to above. Algebraic properties of the inverse limit of a sequence of groups are considered in $\S 4$. A generalization of a result of Milnor [3] is proved asserting that if $X=\bigcup_{i=1}^{\infty} X_{i}$ where $X_{i}$ is closed and $X_{i} \subset$ int $X_{i+1}$ for all $i$ then for an additive cohomology 
theory $H, \delta$ on $X, H(X)$ is related to the inverse limit $\lim \left\{H\left(X_{i}\right)\right\}$ and its derived functor. In $\S 5$ this is applied to the case of additive cohomology on a simplicial complex which is locally constant on every open simplex. It is shown how $H(|K|)$ is related to $\lim \left\{\left(\left|K^{p}\right|\right)\right\}$ and its derived functor. This is used in $\S 6$ to construct the spectral sequence for such cohomology theories. The spectral sequence is then used to prove uniqueness of additive cohomology theories on complexes.

\section{CONTRAVARIANT FUNCTORS ON A SPACE}

In this section we establish some properties of contravariant functors on the category of closed subsets of a topological space. Of particular interest in the sequel are those functors which are locally constant in the sense defined below.

If $H$ is a contravariant functor from $\operatorname{cl}(X)$ to the category of abelian groups and if $A, B \in \operatorname{cl}(X)$ with $A \subset B$ then given $u \in H(B)$ we use $u \mid A$ to denote $H(i)(u) \in H(A)$ where $i: A \rightarrow B$ is the inclusion map, and we also use $\rho$ (or $\left.\rho_{A}\right): H(B) \rightarrow H(A)$ to denote $H(i)$. Given $x \in A$ where $A \in \operatorname{cl}(X)$ an extension of $H(x)$ over $A$ is a homomorphism

$$
\theta: H(x) \rightarrow H(A)
$$

such that

(i) $\theta(u) \mid x=u$ for every $u \in H(x)$, and

(ii) for every $y \in A$, the composite $H(x) \stackrel{\theta}{\rightarrow} H(A) \stackrel{\rho_{y}}{\rightarrow} H(y)$ is an isomorphism.

Remarks. (2.1) If there is a homomorphism $\theta: H(x) \rightarrow H(A)$ such that (ii) above is satisfied, then the composite

$$
H(x) \stackrel{\theta}{\rightarrow} H(A) \stackrel{p_{x}}{\rightarrow} H(x)
$$

is an isomorphism, and if $\theta^{\prime}=\theta \circ\left(\rho_{x} \theta\right)^{-1}: H(x) \rightarrow H(A)$, then $\theta^{\prime}$ satisfies (i) and (ii) so $\theta^{\prime}$ is an extension of $H(x)$ over $A$.

(2.2) If there is an extension $\theta$ of $H(x)$ over $A$ for some $x \in A$, there is an extension of $H(y)$ over $A$ for every $y \in A$. In fact, the composite $\rho_{y} \circ \theta$ is an isomorphism $H(x) \approx H(y)$ and if $\theta_{y}: H(y) \rightarrow H(A)$ is defined by $\theta_{y}=\theta \circ\left(\rho_{y} \circ \theta\right)^{-1}: H(y) \rightarrow H(A)$, then $\rho_{y} \theta_{y}=\left(\rho_{y} \theta\right) \circ\left(\rho_{y} \theta\right)^{-1}=1_{H(y)}$ so (i) is satisfied and, for $z \in A, \rho_{z} \theta_{y}=\left(\rho_{z} \theta\right) \circ\left(\rho_{y} \theta\right)^{-1}$ is the composite of two isomorphisms, so is an isomorphism, showing that (ii) is satisfied.

A closed subset $A \subset X$ is said to be extensive for $H$ if for every $x \in A$ there is an extension of $H(x)$ over $A$. Clearly the empty set $\varnothing$ is extensive for $A$, and if $A$ is nonempty it follows from (2.2) above that $A$ is extensive for $H$ if and only if there is some $x \in A$ such that $H(x)$ has an extension over $A$. $H$ is said to be locally constant if every point of $X$ has a closed neighborhood extensive for $H$.

Remarks. (2.3) If $A^{\prime}$ is a closed subset of $A$ and $A$ is extensive for $H$, then $A^{\prime}$ is extensive for $H$. In fact, if $x \in A^{\prime}$ and $\theta: H(x) \rightarrow H(A)$ is an extension of $H(x)$ over $A$, then $\rho_{A^{\prime}} \theta: H(x) \rightarrow H\left(A^{\prime}\right)$ is easily seen to be an extension of $H(x)$ over $A^{\prime}$.

(2.4) If $H$ is the restriction to $\operatorname{cl}(X)$ of a contravariant functor from the category of all closed subsets of $X$ and all continuous maps between them, then $H$ is locally constant. In fact, let $c: X \rightarrow x$ be the constant map. Then 
$H(c): H(x) \rightarrow H(X)$ is easily verified to be an extension of $H(x)$ over $X$ so $X$ is extensive for $H$ and so $H$ is locally constant.

A contravariant functor $H$ on $\operatorname{cl}(X)$ is continuous if for every closed subset $A$ of $X$ there is an isomorphism

$$
\rho: \lim _{\longrightarrow}\{H(N) \mid N \text { a closed neighborhood of } A \text { in } X\} \approx H(A)
$$

where $\rho\{u\}=u \mid A$ for $u \in H(N)$.

Remarks (2.5). If $H$ is a continuous functor, then for $u, u^{\prime} \in H(A)$ the set $S=\left\{x \in A|u| x=u^{\prime} \mid x\right\}$ is open in $A$ (for if $\left(u-u^{\prime}\right) \mid x=0$ there is a closed neighborhood $N$ of $x$ in $A$ such that $\left.\left(u-u^{\prime}\right) \mid N=0\right)$. If $H$ is also locally constant, the set $T=\left\{x \in X|u| x \neq u^{\prime} \mid x\right\}$ is also open in $A$. For, if $\theta: H(x) \rightarrow H(N)$ is an extension of $H(x)$ to $N$ where $N$ is a closed neighborhood of $x$ in $A$, then $u|x=\theta(u \mid x)| x$ and $u^{\prime}\left|x=\theta\left(u^{\prime} \mid x\right)\right| x$. By continuity of $H$, there is a closed neighborhood $N^{\prime}$ of $x$ in $N$ such that $u\left|N^{\prime}=\theta(u \mid x)\right| N^{\prime}$ and $u^{\prime}\left|N^{\prime}=\theta\left(u^{\prime} \mid x\right)\right| N^{\prime}$. It follows that if $x \in T$ then for $y \in N^{\prime}$,

$$
u\left|y=\left(u \mid N^{\prime}\right)\right| y=\theta(u \mid x)\left|y \neq \theta\left(u^{\prime} \mid x\right)\right| y=\left(u^{\prime} \mid N^{\prime}\right)\left|y=u^{\prime}\right| y,
$$

the inequality because $\rho_{y} \theta$ is an isomorphism from $H(x)$ to $H(y)$. Therefore, $N^{\prime} \subset T$ so $T$ is open in $A$.

(2.6) If $H$ is continuous and locally constant on $\operatorname{cl}(X)$ and $A$ is a connected closed subset of $X$ which is extensive for $H$, then for every $x, y \in A$ there is a canonical isomorphism $h_{x}^{y}: H(x) \approx H(y)$ such that

(a) $h_{x}^{x}=1_{H(x)}$ for $x \in A$,

(b) $h_{x}^{z}=h_{y}^{z} \circ h_{x}^{y}$ for $x, y, z \in A$. First, if $\theta: H\left(x_{0}\right) \rightarrow H(A)$ is an extension of $H\left(x_{0}\right)$ over $A$, we show that $\rho_{x} \theta: H\left(x_{0}\right) \approx H(x)$ is independent of $\theta$. If $\theta^{\prime}: H\left(x_{0}\right) \rightarrow H(A)$ is another extension of $H\left(x_{0}\right)$ over $A$, then by (2.5), for $u \in H\left(x_{0}\right)$ the set $S_{u}=\left\{y \in A|\theta(u)| y=\theta^{\prime}(u) \mid y\right\}$ is open and closed in $A$. Since $x_{0} \in S_{u}$ and $A$ is connected, it follows that $A=S_{u}$. Therefore, $\rho_{x} \theta(u)=\rho_{x} \theta^{\prime}(u)$ for each $x \in A$. Since this is so for every $u \in H\left(x_{0}\right), \rho_{x} \theta=$ $\rho_{x} \theta^{\prime}$. Thus, setting $h_{x}^{y}=\left(\rho_{y} \theta\right) \circ\left(\rho_{x} \theta\right)^{-1}$ we obtain a canonical isomorphism (independent of $\theta$ ) satisfying (a) and (b).

(2.7) Let $H$ be a continuous locally constant contravariant functor on $\operatorname{cl}(X)$. $H$ determines a local system of groups $\mathscr{H}$ on $X$ as follows: Let $\mathscr{H}(x)=H(x)$ for $x \in X$. If $\omega: I \rightarrow X$ is a path in $X$, there is a partition $0=t_{0}<t_{1}<$ $\cdots<t_{m}=1$ of $I$ such that $\omega\left(\left[t_{i-1}, t_{i}\right]\right)$ is a connected closed set which is extensive for $H$ for all $i=1,2, \ldots, m$. By (2.6) there is a canonical isomorphism $h_{i}: H\left(\omega\left(t_{i-1}\right)\right) \approx H\left(\omega\left(t_{i}\right)\right)$ corresponding to the set $\omega\left(\left[t_{i-1}, t_{i}\right]\right)$. Then $h_{\omega}: H(\omega(0)) \approx H(\omega(1))$ is defined to be the composite $h_{m} \circ \cdots \circ h_{2} \circ h_{1}$. The resulting isomorphism is easily seen to be the same for two partitions of $I$ one of which is a subdivision of the other (using (b) of (2.6)). Given any two partitions of $I$ there is a common subdivision, and this shows that $h_{\omega}$ is independent of the choice of the partition. Clearly, if $\omega, \omega^{\prime}$ are paths in $X$ such that $h(1)=h^{\prime}(0)$, then $h_{\omega * \omega^{\prime}}=h_{\omega^{\prime}} \circ h_{\omega}$.

Finally, in case $F: I \times I \rightarrow X$ is a homotopy from $\omega$ to $\omega^{\prime}$, there is a subdivision of $I \times I$ into small rectangles each of which has image under $F$ which is a connected closed set extensive for $H$. Chasing the isomorphisms around these small rectangles we see that $h_{\omega}=h_{\omega^{\prime}} a$. Thus, we have a local system $\mathscr{H}, h$ on $X$. 
(2.8) If $H$ is the restriction to $\operatorname{cl}(X)$ of a contravariant functor from the category of closed subsets of $X$ and continuous maps between them, the local system $\mathscr{H}$ determined by $H$ as in (2.7) above is a simple system. This follows from (2.6) and the fact that by (2.4) $X$ is extensive for $H$.

\section{LOCALLY CONSTANT COHOMOLOGY THEORIES}

In this section we recall the definition of a cohomology theory on a space and study those which are locally constant. A main result is the homotopy property for nonnegative cohomology theories which are additive or weakly additive on a category of paracompact spaces.

A cohomology theory $H, \delta$ on a space $X$ consists of:

(i) a contravariant functor $H$ from $\operatorname{cl}(X)$ to the category of graded abelian groups such that $H(\varnothing)=0$, and

(ii) a natural transformation of degree 1, $\delta: H(A \cap B) \rightarrow H(A \cup B)$ for every $A, B \in \operatorname{cl}(X)$

such that $H$ is continuous (i.e. for every $q, H^{q}$ is a continuous functor to the category of abelian groups as defined in $\S 2$ ) and such that for every $A$, $B \in \operatorname{cl}(X)$ there is an exact Mayer-Vietoris sequence

$$
\cdots \stackrel{\beta}{\rightarrow} H^{q-1}(A \cap B) \stackrel{\delta}{\rightarrow} H^{q}(A \cup B) \stackrel{\alpha}{\rightarrow} H^{q}(A) \oplus H^{q}(B) \stackrel{\beta}{\rightarrow} H^{q}(A \cap B) \stackrel{\delta}{\rightarrow} \cdots
$$

where $\alpha(u)=(u|A, u| B)$ and $\beta(u, v)=u|A \cap B-v| A \cap B \mid$.

The cohomology theory is nonnegative if $H^{q}(A)=0$ for $q<0$ and all $A \in \operatorname{cl}(X)$. It is additive (or weakly additive) if given a discrete family $\left\{A_{j}\right\}_{j \in J}$ in $\operatorname{cl}(X)$ (discrete means that every point of $X$ has a neighborhood meeting at most one member of the family) there is an isomorphism

$$
\sigma: H^{q}\left(\bigcup_{j \in J} A_{j}\right) \approx \prod_{j \in J} H^{q}\left(A_{j}\right)
$$

where $\sigma(u)=\left\{u \mid A_{j}\right\}_{j \in J}$ for $u \in H^{q}\left(\bigcup_{j \in J} A_{j}\right)$ (or there is an isomorphism $\imath: \bigoplus_{j \in J} H^{q}\left(A_{j}\right) \approx H^{q}\left(\bigcup_{j \in J} A_{j}\right)$ where $l \mid H^{q}\left(A_{k}\right)$ is the composite

$$
\left.H^{q}\left(A_{k}\right) \subset H^{q}\left(A_{k}\right) \oplus H^{q}\left(\bigcup_{j \neq k} A_{j}\right) \approx H^{q}\left(\bigcup_{j \in J} A_{j}\right)\right) .
$$

A cohomology theory is locally constant if, for every $q, H^{q}$ is a locally constant functor as defined in $\S 2$.

Example. Let $f: Y \rightarrow X$ be a fiber bundle with compact fiber (e.g. $Y$ is locally the product of $X$ with some fixed compact space $F$ ) and define $H, \delta$ on $X$ so that $H(A)=$ Cech cohomology of $f^{-1}(A)$ (with some fixed coefficient group) and $\delta$ is suitably defined. This is a nonnegative additive cohomology theory which is locally constant on $X$ (the compactness of $F$ implies that if $A$ is closed in $X$ and $N$ is a closed neighborhood of $f^{-1}(A)$ in $Y$ there is a closed neighborhood $B$ of $A$ in $X$ such that $f^{-1}(B) \subset N$ and this is used in proving $H$ is continuous on $X$ ).

Proposition (3.1). Let $H, \delta$ be a locally constant cohomology theory on the closed unit interval $I$. The $\rho_{x}: H(I) \rightarrow H(x)$ is an isomorphism for every $x \in I$. 
Proof. We show that $\rho_{x}$ is an epimorphism. Because $H$ is locally constant there exists a partition $0=t_{0}<t_{1}<\cdots<t_{m}=1$ of $I$ such that for each $i=1,2, \ldots, m$ there is an extension $\theta_{i}$ of $H\left(t_{i}\right)$ over $\left[t_{i-1}, t_{i}\right]$. Assume $x \in$ $\left[t_{i-1}, t_{j}\right]$ for some $i \leq j$ and that $H\left(\left[t_{i-1}, t_{j}\right]\right) \rightarrow H(x)$ is an epimorphism. If $i-1>0($ or $j<m)$ we prove that $H\left(\left[t_{i-2}, t_{j}\right]\right) \rightarrow H(x)\left(\right.$ or $H\left(\left[t_{i-1}, t_{j+1}\right]\right) \rightarrow$ $H(x))$ is also an epimorphism. The proofs in the two cases are similar so we only treat the former case.

There is an exact sequence

$$
H\left(\left[t_{i-2}, t_{j}\right]\right) \stackrel{\alpha}{\rightarrow} H\left(\left[t_{i-2}, t_{i-1}\right]\right) \oplus H\left(\left[t_{i-1}, t_{j}\right]\right) \stackrel{\beta}{\rightarrow} H\left(t_{i-1}\right) .
$$

Given $u \in H(x)$ let $u^{\prime} \in H\left(\left[t_{i-1}, t_{j}\right]\right)$ be such that $u^{\prime} \mid x=u$. Then

$$
\left(\theta_{i-1}\left(u^{\prime} \mid t_{i-1}\right), u^{\prime}\right) \in H\left(\left[t_{i-2}, t_{i-1}\right]\right) \oplus H\left(\left[t_{i-1}, t_{j}\right]\right)
$$

is in the kernel of $\beta$ so there is $u^{\prime \prime} \in H\left(\left[t_{i-2}, t_{j}\right]\right)$ such that

$$
\alpha\left(u^{\prime \prime}\right)=\left(\theta_{i-1}\left(u^{\prime} \mid t_{i-1}\right), u^{\prime}\right) .
$$

Then $u^{\prime \prime}\left|x=\left(u^{\prime \prime} \mid\left[t_{i-1}, t_{j}\right]\right)\right| x=u^{\prime} \mid x=u$ proving that $H\left(\left[t_{i-2}, t_{j}\right]\right) \rightarrow H(x)$ is an epimorphism. By a straightforward induction it follows that

$$
H(I)=H\left(\left[t_{0}, t_{m}\right]\right) \rightarrow H(x)
$$

is an epimorphism.

We show $\rho_{x}$ is a monomorphism. Assume $\rho_{x}(u)=0$ for some $u \in H(I)$. By (2.5) it follows that $u \mid y=0$ for all $y \in I$. Since $H$ is continuous, there is a partition $0=t_{0}<t_{1}<\cdots<t_{m}=1$ of $I$ such that $u \mid\left[t_{i-1}, t_{i}\right]=0$ for $i=1,2, \ldots, m$. We prove by induction on $I$ that $u \mid\left[0, t_{i}\right]=0$. This is true for $i=1$ by choice of the interval $\left[0, t_{1}\right]=0$. Assume $i>0$ and that $u \mid\left[0, t_{i-1}\right]=0$. There is an exact sequence

$$
\begin{aligned}
H\left(\left[0, t_{i-1}\right]\right) & \oplus H\left(\left[t_{i-1}, t_{i}\right]\right) \stackrel{\beta}{\rightarrow} H\left(t_{i-1}\right) \stackrel{\delta}{\rightarrow} H\left(\left[0, t_{i}\right]\right) \\
& \stackrel{\leftrightarrow}{\rightarrow} H\left(\left[0, t_{i-1}\right]\right) \oplus H\left(\left[t_{i-1}, t_{i}\right]\right) .
\end{aligned}
$$

Since $\alpha\left(u \mid\left[0, t_{i}\right]\right)=\left(u\left|\left[0, t_{i-1}\right], u\right|\left[t_{i-1}, t_{i}\right]\right)=(0,0)$, there is $v \in H\left(t_{i-1}\right)$ such that $\delta_{v}=u \mid\left[0, t_{i}\right]$. It follows from the first part of the proof above that $\beta$ is an epimorphism so $v=\beta w$ for some $w$ and $u \mid\left[0, t_{i}\right]=\delta v=$ $\delta \beta w=0$ completing the induction. Hence $u=u \mid\left[0, t_{m}\right]=0$ so $\rho_{x}$ is a monomorphism.

In the future all cohomology theories $H, \delta$ on a space $X$ will be assumed to be additive or weakly additive (in the case $X$ is compact every cohomology theory is both additive and weakly additive). If $A$ is a paracompact subset of $X$ we say $H, \delta$ is of finite type on $A$ if either $H$ is nonnegative or $A$ is locally finite dimensional (i.e. every point of $A$ has a closed finite dimensional neighborhood). The hypothesis of finite type implies that a comparison theorem is valid for two cohomology theories on $A$ constructed from $H, \delta$ as in the following.

Corollary (3.2). Let $H, \delta$ be a cohomology theory on $X$ and assume $A$ is a space such that $A \times I$ is a closed subset of $X$ and $H$ is locally constant on $a \times I$ 
for each $a \in A$. If $H, \delta$ is of finite type on $A$, then $\rho: H(A \times I) \rightarrow H(A \times t)$ is an isomorphism for every $t \in I$.

Proof. Let $t \in I$ be fixed and define two cohomology theories $H^{\prime}, \delta^{\prime}$ and $H^{\prime \prime}, \delta^{\prime \prime}$ on $A$ by $H^{\prime}(B)=H(B \times I)$ and $H^{\prime \prime}(B)=H(B \times t)$ for $B$ closed in $A\left(\delta^{\prime}, \delta^{\prime \prime}\right.$ suitably defined in each case). The restriction map $\rho: H(B \times I) \rightarrow$ $H(B \times t)$ defines a homomorphism of cohomology theories from $H^{\prime}, \delta^{\prime}$ to $H^{\prime \prime}$, $\delta^{\prime \prime}$. By Proposition (3.1) this homomorphism is an isomorphism of $H^{\prime}(a)$ with $H^{\prime \prime}(a)$ for every $a \in A$. If $H$ is additive or weakly additive the same is true for both $H^{\prime}$ and $H^{\prime \prime}$. In case $H$ is nonnegative so are $H^{\prime}$ and $H^{\prime \prime}$ and the result follows from the theorem in [8]. In case $A$ is locally finite dimensional, the result follows similarly from the theorem in [8].

Corollary (3.3). Let $H, \delta$ be a locally constant cohomology theory on $I^{n}$. Then $\rho_{x}: H\left(I^{n}\right) \approx H(x)$ is an isomorphism for every $x \in I^{n}$.

Proof. We use induction on $n$. For $n=1$ the result is Proposition (3.1). Assume the result is valid for $n-1$ and that $H$ is locally constant on $I^{n}$. Let $A=I^{n-1}$ and note that $I^{n}=A \times I$. Since $I^{n}$ is compact, $H$ is additive and since $I^{n-1}$ has finite dimension $H, \delta$ is of finite type on $I^{n-1}$. By Corollary (3.2), $H\left(I^{n}\right) \rightarrow H\left(I^{n-1} \times t\right)$ is an isomorphism for every $t \in I$. By the induction hypothesis, $H\left(I^{n} \times t\right) \rightarrow H(y, t)$ is an isomorphism for every $y \in I^{n-1}$. Combining these two isomorphisms we obtain $\rho_{x}: H\left(I^{n}\right) \rightarrow H(x)$ is an isomorphism for every $x \in I^{n}$ (since $x=(y, t)$ for some $y \in I^{n-1}$, $t \in I)$.

Let $\mathscr{C}$ be a category of spaces and continuous maps such that for every object $X \in \mathscr{C}, \operatorname{cl}(X)$ is a subcategory of $\mathscr{C}$. A cohomology theory $H, \delta$ on $\mathscr{C}$ [6, 7] consists of:

(i) a contravariant functor $H$ from $\mathscr{C}$ to the category of graded abelian groups such that $H(\varnothing)=0$, and

(ii) for every closed triad $(X ; A, B)$ with $X \in \mathscr{C}, A, B \subset X$ a natural transformation $\delta: H(A \cap B) \rightarrow H(A \cup B)$ of degree 1 , such that for every $X \in \mathscr{C}$ the restriction of $H, \delta$ to $\operatorname{cl}(X)$ is a cohomology theory on $X$.

The cohomology theory is nonnegative, weakly additive, or additive, respectively, if its restriction to $\operatorname{cl}(X)$ has the corresponding property for every $X \in$ $\mathscr{C}$. A cohomology theory on $\mathscr{C}$ is invariant under homotopy if for every $f_{0}, f_{1}: X \rightarrow Y$ which are homotopic in $\mathscr{C}$ (i.e. there is a continuous map $F: X \times I \rightarrow Y$ in $\mathscr{C}$ such that $F(x, 0)=f_{0}(x), F(x, 1)=f_{1}(x)$ for all $x \in X)$ then $H\left(f_{0}\right)=H\left(f_{1}\right): H(X) \rightarrow H(Y)$.

The following is a direct consequence of Corollary (3.2).

Theorem (3.4). Let $H, \delta$ be a nonnegative cohomology theory on a category $\mathscr{C}$ of paracompact spaces such that $H$ is additive or weakly additive. Then $H$ is invariant under homotopy.

Our final result is

Theorem (3.5). Let $H, \delta$ be a cohomology theory on $[0,1]$ which is locally constant on $(0,1]$. Then $H([0,1]) \rightarrow H(\{0\})$ is an isomorphism. 
Proof. For $a$ with $0<a<1$ there is an exact sequence

$$
\cdots \rightarrow H([0, a]) \oplus H([a, 1]) \stackrel{\beta}{\rightarrow} H(\{a\}) \stackrel{\delta}{\rightarrow} H([0,1]) \stackrel{\alpha}{\rightarrow} H([0, a]) \oplus H([a, 1]) .
$$

Because $H, \delta$ is locally constant on $[a, 1]$ by Proposition (3.1) there is also an exact sequence

$$
\cdots \rightarrow 0 \rightarrow H([a, 1]) \rightarrow H(\{a\}) \rightarrow 0 \rightarrow \cdots .
$$

The quotient of the first by the second is the exact sequence

$$
H([0, a]) \rightarrow 0 \rightarrow H([0,1]) \rightarrow H([0, a]) \rightarrow \cdots .
$$

Therefore, $\rho: H([0,1]) \approx H([0, a])$. Taking the direct limit as $a \rightarrow 0$ we obtain

$$
H([0,1]) \approx \underset{a \rightarrow 0}{\lim }\{H([0, a])\} \approx H(\{0\})
$$

the last isomorphism by the continuity of $H$.

\section{A LIMIT PROPERTY OF COHOMOLOGY}

Let $X$ be a space which is the increasing union of a sequence of closed subspaces

$$
X_{1} \subset X_{2} \subset \cdots
$$

such that $X=\bigcup X_{i}$. If $H, \delta$ is a cohomology theory on $X$, there is a restriction map $H(X) \rightarrow H\left(X_{i}\right)$ for each $i$, and we shall use these maps to compare $H(X)$ with the inverse $\operatorname{limit} \lim \left\{H\left(X_{i}\right)\right\}$ of the sequence $\left\{H\left(X_{i}\right)\right\}$. We begin with a review of some relevant algebra.

An inverse sequence [4] of abelian groups is a sequence $\left\{G_{i}\right\}_{i=1,2, \ldots}$ of abelian groups together with homomorphisms $p: G_{i+1} \rightarrow G_{i}$ for each $i$. Let $C\left\{G_{i}\right\}$ be the cochain complex all of whose cochain groups are 0 except for $C^{0}=\prod_{i} G_{i}$ and $C^{1}=\prod_{i} G_{i}$ and with coboundary $d: C^{0} \rightarrow C^{1}$ defined by $d\left(g_{1}, g_{2}, \ldots\right)$ $=\left(g_{1}-p g_{2}, g_{2}-p g_{3}, \ldots\right)$. The inverse limit $\lim _{4}\left\{G_{i}\right\}$ of the sequence $\left\{G_{i}\right\}$ is defined to equal $H^{0}\left(C\left\{G_{i}\right\}\right)=\operatorname{ker}\left(d: C^{0} \rightarrow C^{1}\right)$ and the derived functor of the inverse limit $\lim ^{1}\left\{G_{i}\right\}$ is defined to equal $H^{1}\left(C\left\{G_{i}\right\}\right)=\operatorname{coker}\left(d: C^{0} \rightarrow C^{1}\right)$.

Remarks. (4.1) Let $A_{1}, A_{2}, \ldots$ be a sequence of abelian groups. Let $G_{i}=$ $A_{1} \times \cdots \times A_{i}$ and let $p: G_{i+1} \rightarrow G_{i}$ be the canonical projection of an $(i+1)$-fold product onto the product of its first $i$ factors. A straightforward calculation shows that

$$
\lim _{\longleftarrow}\left\{G_{i}\right\}=\left\{a_{1},\left(a_{1}, a_{2}\right),\left(a_{1}, a_{2}, a_{3}\right), \ldots \mid a_{i} \in A_{i}\right\} \approx \prod_{i} A_{i}
$$

and $\lim ^{1}\left\{G_{i}\right\}=0$.

(4.2) Assume $p: G_{i+1} \rightarrow G_{i}$ is an isomorphism for all $i \geq n$. Then it can be verified that $\lim _{\longleftarrow}\left\{G_{i}\right\} \approx G_{n}$ and $\lim ^{1}\left\{G_{i}\right\}=0$.

(4.3) If $p: G_{i+1} \rightarrow G_{i}$ is an epimorphism for each $i$, then it is easy to see that $\lim ^{1}\left\{G_{i}\right\}=0$.

(4.4) If $0 \rightarrow G_{i}^{\prime} \stackrel{\phi}{\rightarrow} G_{i} \stackrel{\psi}{\rightarrow} G_{i}^{\prime \prime} \rightarrow 0, i=1,2, \ldots$, is an inverse sequence of short exact sequences, there is a six-term exact sequence

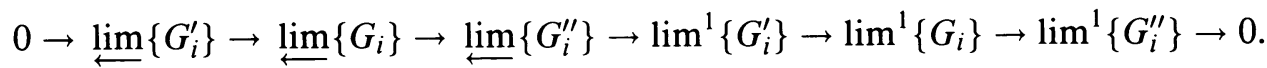


Lemma (4.5). Let $G_{i} \stackrel{\phi_{i}}{\rightarrow} H_{i}$ be an inverse sequence of homomorphisms and suppose $\lim ^{1}\left\{G_{i}\right\}=0=\lim ^{1}\left\{H_{i}\right\}$. For the induced map $\stackrel{\lim }{\longleftarrow}\left\{G_{i}\right\} \stackrel{\phi}{\rightarrow} \underset{\lim }{\longleftarrow}\left\{H_{i}\right\}$ there is an isomorphism

$$
\operatorname{ker} \phi \approx \lim _{\longleftarrow}\left\{\operatorname{ker} \phi_{i}\right\}
$$

and a short exact sequence

$$
0 \rightarrow \lim ^{1}\left\{\operatorname{ker} \phi_{i}\right\} \rightarrow \operatorname{coker} \phi \rightarrow \underset{\lim }{\longleftarrow}\left\{\operatorname{coker} \phi_{i}\right\} \rightarrow 0 .
$$

Proof. Let $C^{i}$ be the chain complex with $C_{1}^{i}=G_{i}, C_{0}^{i}=H_{i}, C_{j}^{i}=0, j \neq$ 0,1 , and $\partial: C_{1}^{i} \rightarrow C_{0}^{i}$ equal to $\phi_{i}: G_{i} \rightarrow H_{i}$. Let $C=\lim \left\{C^{i}\right\}$. Then $H_{1}(C)=\operatorname{ker} \phi$ and $H_{0}(C)=\operatorname{coker} \phi$. The hypothesis $\lim ^{1}\left\{G_{i}\right\}=0=\lim ^{1}\left\{H_{i}\right\}$ implies $\lim ^{1}\left\{C^{i}\right\}=0$. By Theorem A.19 of [4] there are short exact sequences

$$
0 \rightarrow \lim ^{1}\left\{H_{2}\left(C^{i}\right)\right\} \rightarrow H_{1}(C) \rightarrow \underset{\lim }{\longleftarrow}\left\{H_{1}\left(C^{i}\right)\right\} \rightarrow 0
$$

and

$$
0 \rightarrow \lim ^{1}\left\{H_{1}\left(C^{i}\right)\right\} \rightarrow H_{0}(C) \rightarrow \underset{\lim }{\longleftarrow}\left\{H_{0}\left(C^{i}\right)\right\} \rightarrow 0 .
$$

The first of these gives $\operatorname{ker} \phi \approx \lim \left\{\operatorname{ker} \phi_{i}\right\}$ and the second gives the short exact sequence

$$
0 \rightarrow \lim ^{1}\left\{\operatorname{ker} \phi_{i}\right\} \rightarrow \operatorname{coker} \phi \rightarrow \underset{\lim }{\longleftarrow}\left\{\operatorname{coker} \phi_{i}\right\} \rightarrow 0 .
$$

We now apply these ideas to the topological situation. Theorem (4.6) below was first proved by Milnor [3] for additive cohomology theories satisfying the Eilenberg-Steenrod axioms on a $\mathrm{CW}$ complex filtered by subcomplexes.

Theorem (4.6). Let $H, \delta$ be an additive cohomology theory on a space $X=\bigcup X_{i}$ where $X_{i}$ is closed in $X$ and $X_{i} \subset$ int $X_{i+1}$ for each $i$. Then there is a short exact sequence for each $q$,

$$
0 \rightarrow \lim ^{1}\left\{H^{q-1}\left(X_{i}\right)\right\} \rightarrow H^{q}(X) \rightarrow \underset{\lim }{\longleftarrow}\left\{H^{q}\left(X_{i}\right)\right\} \cdots \rightarrow 0 .
$$

Proof. Let $A=\bigcup_{i \text { odd }}\left(X_{i}-\operatorname{int} X_{i-1}\right)$ and $B=\bigcup_{i \text { even }}\left(X_{i}-\operatorname{int} X_{i-1}\right)$ (where we take $\left.X_{0}=\varnothing\right)$. Then $A$ is a discrete union of the closed sets $\left\{X_{i}-\text { int } X_{i-1}\right\}_{i \text { odd }}$ and $B$ is a discrete union of the closed sets $\left\{X_{i}-\operatorname{int} X_{i-1}\right\}_{i}$ even (because if $x \in X$ and $j$ is the smallest index such that $x \in$ int $X_{j}$, then $x \notin X_{j-2}$ so that int $X_{j}-X_{j-2}$ is an open set about $x$ that can only meet $X_{j}-$ int $X_{j-1}$ and $X_{j-1}-$ int $X_{j-2}$ and one of these is in $A$ while the other is in $B$ ). Furthermore, $X=A \cup B$ and $A \cap B=\bigcup_{i}\left(X_{i}-\operatorname{int} X_{i-1}\right)$. Therefore, there is an exact sequence

$$
\begin{aligned}
\cdots & \rightarrow H^{q-1}(A) \oplus H^{q-1}(B) \stackrel{\beta^{\prime}}{\rightarrow} H^{q-1}(A \cap B) \stackrel{\delta}{\rightarrow} H^{q}(X) \\
& \rightarrow H^{q}(A) \oplus H^{q}(B) \stackrel{\beta}{\rightarrow} H^{q}(A \cap B) \rightarrow \cdots .
\end{aligned}
$$

For each $j$ let

$$
A_{j}=\bigcup_{\substack{i \leq j \\ i \text { odd }}}\left(X_{i}-\operatorname{int} X_{i-1}\right), \quad B_{j}=\bigcup_{\substack{i \leq j \\ i \text { even }}}\left(X_{i}-\operatorname{int} X_{i-1}\right)
$$

and note that there is a similar exact sequence

$$
\begin{aligned}
\cdots & \rightarrow H^{q-1}\left(A_{j}\right) \oplus H^{q-1}\left(B_{j}\right) \stackrel{\beta_{j}^{\prime}}{\rightarrow} H^{q-1}\left(A_{j} \cap B_{j}\right) \stackrel{\delta_{j}}{\rightarrow} H^{q}\left(X_{j}\right) \\
& \rightarrow H^{q}\left(A_{j}\right) \oplus H^{q}\left(B_{j}\right) \stackrel{\beta_{j}}{\rightarrow} H^{q}\left(A_{j} \cap B_{j}\right) \rightarrow \cdots .
\end{aligned}
$$


As $j$ varies these latter sequences form an inverse sequence of exact sequences and the restriction maps map the former sequence into each term of this inverse sequence. Furthermore, by (4.1)

$$
H^{q}(A) \oplus H^{q}(B) \approx \lim _{\longleftarrow}\left\{H^{q}\left(A_{j}\right) \oplus H^{q}\left(B_{j}\right)\right\}
$$

and

$$
H^{q}(A \cap B) \approx \lim _{\longleftarrow}\left\{H^{q}\left(A_{j} \cap B_{j}\right)\right\}
$$

and under these isomorphisms $\beta$ corresponds to $\lim _{\longleftarrow}\left\{\beta_{j}\right\}$ and $\beta^{\prime}$ corresponds to $\lim _{\longleftarrow}\left\{\beta_{j}^{\prime}\right\}$. Also $\lim ^{1}\left\{H^{q}\left(A_{j}\right) \oplus H^{q}\left(B_{j}\right)\right\}=0=\lim ^{1}\left\{H^{q}\left(A_{j} \cap B_{j}\right)\right\}$, and there is a short exact sequence

$$
0 \rightarrow \operatorname{coker} \beta^{\prime} \rightarrow H^{q}(X) \rightarrow \operatorname{ker} \beta \rightarrow 0
$$

mapped into the inverse sequence of short exact sequences

$$
0 \rightarrow \operatorname{coker} \beta_{j}^{\prime} \rightarrow H^{q}\left(X_{j}\right) \rightarrow \operatorname{ker} \beta_{j} \rightarrow 0 .
$$

Since coker $\beta_{j}^{\prime}$ is a quotient of $H^{q-1}\left(A_{j} \cap B_{j}\right)$ and $\lim ^{1}\left\{H^{q-1}\left(A_{j} \cap B_{j}\right)\right\}=0$, it follows from (4.4) that $\lim ^{1}\left\{\operatorname{coker} \beta_{j}^{\prime}\right\}=0$. Therefore, (4.4) applied to the inverse sequence of short exact sequences above yields a short exact sequence

$$
0 \rightarrow \lim _{\longleftarrow}\left\{\operatorname{coker} \beta_{j}^{\prime}\right\} \rightarrow \underset{\lim }{\longleftarrow}\left\{H^{q}\left(X_{j}\right)\right\} \rightarrow \underset{\lim }{\longleftarrow}\left\{\operatorname{ker} \beta_{j}\right\} \rightarrow 0
$$

and there is a commutative diagram whose rows are exact

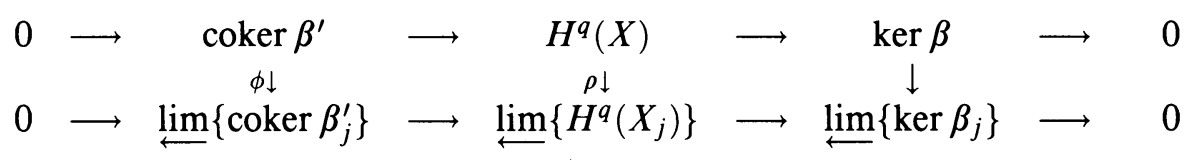

By the first part of Lemma (4.5) the right-hand vertical map is an isomorphism, and by the second part of Lemma (4.5) the left-hand vertical map is an epimorphism whose kernel is isomorphic to $\lim ^{1}\left\{\operatorname{ker} \beta_{j}^{\prime}\right\}$. If follows that $\rho$ is an epimorphism and $\operatorname{ker} \rho \approx \operatorname{ker} \varphi \approx \lim ^{1}\left\{\operatorname{ker} \beta_{j}^{\prime}\right\}$.

To reinterpret the latter group note that there is an inverse sequence of short exact sequences

$$
0 \rightarrow D_{j} \rightarrow H^{q-1}\left(X_{j}\right) \rightarrow \operatorname{ker} \beta_{j}^{\prime} \rightarrow 0
$$

where $D_{j}$ is a quotient of $H^{q-2}\left(A_{j} \cap B_{j}\right)$ so $\lim ^{1}\left\{D_{j}\right\}=0$. This implies that $\lim ^{1}\left\{H^{q-1}\left(X_{j}\right)\right\} \approx \lim ^{1}\left\{\operatorname{ker} \beta_{j}^{\prime}\right\}$ so that $\rho: H^{q}(X) \rightarrow \underset{\lim }{\longleftarrow}\left\{H^{q}\left(X_{j}\right)\right\}$ is an epimorphism whose kernel is isomorphic to $\lim ^{1}\left\{H^{q-1}\left(X_{j}\right)\right\}$.

\section{LOCALLY CONSTANT COHOMOLOGY ON POLYHEDRA}

In this section we begin the study of cohomology theories on polyhedra (a polyhedron is the space of a simplicial complex) which are locally constant on every open simplex. It is clear that if a cohomology theory is locally constant on every open simplex of $|K|$ it is also locally constant on every open simplex of $\left|K^{\prime}\right|$ for every subdivision $K^{\prime}$ of $K$. 
Examples. (5.1) Let $f: X \rightarrow Y$ be a closed simplicial map of one polyhedron into another. Then $f_{*} H$ defined on $Y$ by $\left(f_{*} H\right)(B)=H\left(f^{-1}(B)\right.$ ) (Čech cohomology with some fixed coefficient group) for $B$ closed in $Y$ is locally constant on every open simplex of $Y$.

(5.2) Let $p: E \rightarrow B$ be a closed projection of a fibration where $B$ is a polyhedron. Then $p_{*} H$ defined as in (5.1) above is locally constant on every open simplex of $B$.

(5.3) Let $X$ be a polyhedron and define ${ }^{\Delta} H$ on $X$ by

$$
{ }^{\Delta} H^{q}(A)=H_{-q}(X, X-A)
$$

(singular homology with some fixed coefficient group) for closed $A \subset X$. Then ${ }^{\Delta} H$ is locally constant on every open simplex of $X$.

Let $X$ be the join of a vertex $v$ with a simplicial complex $Y$ and topologize both $X$ and $Y$ by the weak topology with respect to their finite subcomplexes. Every point of $X$ has the form $t v+(1-t) y$ for some $y \in Y$ and $0 \leq t \leq 1$.

Lemma (5.4). If $U$ is any open set in $|X|$ containing $v$ there is a continuous function $f:|Y| \rightarrow(0,1]$ such that for every $y \in|Y|$ the set $\{t v+(1-t) y \mid 0 \leq$ $t \leq f(y)\} \subset \dot{U}$.

Proof. $f$ will be constructed inductively over the successive skeleta of $Y$. It is clear that $f^{0}:\left|Y^{0}\right| \rightarrow(0,1]$ can be defined with the given property. Assume $f^{k}:\left|Y^{k}\right| \rightarrow(0,1]$ satisfies the condition for $y \in\left|Y^{k}\right|$ and let $s$ be a $(k+1)$-simplex of $Y$. Then $f^{k}$ is already defined on $|\dot{s}|$. Introduce "polar coordinates" $\left(y, t^{\prime}\right)$ in $|s|$ where $y \in|\dot{s}|, 0 \leq t^{\prime} \leq 1$ and $(y, 1)=y,(y, 0)=$ barycenter of $s$. Because $U$ is open and

$$
A=\left\{t v+(1-t) y|y \in| \dot{s} \mid, 0 \leq t \leq f^{k}(y)\right\} \subset U
$$

it follows from the compactness of $A$ that there is $0<\varepsilon<1$ such that $\{t v+$ $\left.(1-t)\left(y, t^{\prime}\right)|y \in| \dot{s} \mid, \varepsilon \leq t^{\prime} \leq 1,0 \leq t \leq f^{k}(y)\right\} \subset U$. Choose $a$ with $0<a \leq 1$ such that

$$
\left\{t v+(1-t)\left(y, t^{\prime}\right)\left|\left(y, t^{\prime}\right) \in\right| \dot{s} \mid, 0 \leq t \leq a\right\} \subset U .
$$

Such a choice of $a$ is possible because every point of $|v s|-U$ has a unique representation in the form $t v+(1-t)\left(y, t^{\prime}\right)$ with $\left(y, t^{\prime}\right) \in|s|$ and $t<1$. Projection of this point to $1-t$ is a continuous function from the compact set $|v s|-U$ to $(0,1]$ so attains a minimum. Any positive $a$ smaller than this minimum will have the desired property.

We now extend $f^{k}$ to $f_{s}:|s| \rightarrow(0,1]$ by

$$
f_{s}\left(y, t^{\prime}\right)= \begin{cases}\frac{t^{\prime}-\varepsilon}{1-\varepsilon} f^{k}(y)+\frac{1-t^{\prime}}{1-\varepsilon} a, & \varepsilon \leq t^{\prime} \leq 1, \\ a, & 0 \leq t^{\prime} \leq \varepsilon .\end{cases}
$$

Then $f_{s}$ is continuous from $|s|$ to $(0,1]$ and $f_{s}(y, 1)=f^{k}(y)$ so $f_{s}$ extends $f^{k}|| \dot{s} \mid$. We verify that $f_{s}$ has the desired property on $|s|$. If $\left(y, t^{\prime}\right) \in|s|$ and $0 \leq t \leq f_{s}\left(y, t^{\prime}\right)$, then in case $t^{\prime} \leq \varepsilon$ we have $t \leq a$ so, by the choice of $a$, $t v+(1-t)\left(y, t^{\prime}\right) \in U$. In case $\varepsilon \leq t^{\prime} \leq 1$, then

$$
f_{s}\left(y, t^{\prime}\right)=\frac{t^{\prime}-\varepsilon}{1-\varepsilon} f^{k}(y)+\frac{1-t^{\prime}}{1-\varepsilon} a \leq \max \left(f^{k}(y), a\right)
$$


so $0 \leq t \leq f_{s}\left(y, t^{\prime}\right) \Rightarrow 0 \leq t \leq \max \left(f^{k}(y), a\right)$ in which case $t v+(1-t)\left(y, t^{\prime}\right) \in$ $U$ by choice of $\varepsilon$ and $a$.

Therefore, we have constructed a continuous $f_{s}:|s| \rightarrow(0,1]$ extending $f^{k}|| \dot{s} \mid$ with the desired property. The collection $\left\{f_{s}\right\}$ for all $s$ of dimension $k+$ 1 fit together to define a continuous function $f^{k+1}:\left|Y^{k+1}\right| \rightarrow(0,1]$ extending $f^{k}$ and having the requisite property.

Then the collection $\left\{f^{k}:\left|Y^{k}\right| \rightarrow(0,1]\right\}_{k=0,1, \ldots}$ determines a continuous map $f:|Y| \rightarrow(0,1]$ with the desired property.

Given a continuous function $f:|Y| \rightarrow(0,1]$ let $N_{f}=\{t v+(1-t) y \mid y \in$ $|Y|, 0 \leq t \leq f(y)\}$. Then $N_{f}$ is a closed neighborhood of $v$ in $|X|$ and, by Lemma 5.4, every neighborhood of $v$ in $|X|$ contains $N_{f}$ for some continuous $f$.

Theorem (5.5). Let $H, \delta$ be a cohomology theory on $|v Y|$ such that for each $y \in|Y|, H$ is locally constant on $(v, y]$. If $f:|Y| \rightarrow(0,1]$ is a continuous function and $H, \delta$ is of finite type on $|v Y|$, then $\rho: H(|v Y|) \rightarrow H\left(N_{f}\right)$ is an isomorphism.

Proof. Let $A=\{t v+(1-t) y|y \in| Y \mid, f(y) \leq t \leq 1\}$. Then $A$ is closed and $A \cap N_{f}=\{f(y) v+(1-f(t)) y|y \in| Y \mid\}$. We show $\rho^{\prime}: H(A) \rightarrow H\left(A \cap N_{f}\right)$ is an isomorphism. There are two cohomology theories on $|Y|$ defined by

$$
H_{1}(B)=H\{t v+(1-t) y \mid y \in B, f(y) \leq t \leq 1\}
$$

and

$$
H_{2}(B)=H\{f(y) v+(1-f(y)) y \mid y \in B\}
$$

for $B$ closed in $Y$ and the restriction map is a homomorphism $H_{1} \rightarrow H_{2}$. Since $H$ is locally constant on $\{t y+(1-t) y \mid f(y) \leq t \leq 1\}$ it follows from Proposition (3.1) that $H_{1}(\{y\}) \rightarrow H_{2}(\{y\})$ is and isomorphism for each $y$. From the hypothesis that $H, \delta$ is of finite type on $|v Y|$ it follows that $H_{1}(B) \approx H_{2}(B)$ for all closed $B \subset Y$. In particular, $H(A)=H_{1}(|Y|) \approx$ $H_{2}(|Y|)=H\left(A \cap N_{f}\right)$. From this and the $M V$ exactness for $N_{f}, A$ we find that $H(|v Y|)=H\left(A \cup N_{f}\right) \approx H\left(N_{f}\right)$.

Corollary (5.6). If $H, \delta$ is a cohomology theory on $|v Y|$ such that for $y \in Y$, $H$ is locally constant on $(v, y]$ and if $H, \delta$ is of finite type on $|v Y|$ then $H(|v Y|) \rightarrow H(\{v\})$ is an isomorphism.

Proof. By Lemma (5.4) the closed neighborhoods $N_{f}$ are cofinal in the family of all neighborhoods of $v$ in $|v Y|$. Therefore, $\lim \left\{H\left(N_{f}\right)\right\} \approx H(\{v\})$. From Theorem (5.5) we have $H(|v Y|) \approx \lim _{\longrightarrow}\left\{H\left(N_{f}\right)\right\}$ and the result follows on combining these isomorphisms.

Let $L$ be a full subcomplex of a simplicial complex $K$ and let $b_{L}:|K| \rightarrow$ $[0,1]$ be the continuous function such that $b_{L}(x)$ is the sum of the barycentric coordinates of $x$ corresponding to vertices in $L$. Then $x \in|L| \Leftrightarrow b_{L}(x)=1$. Given $0<a<1$ let $N_{a}(|L|)=\left\{x \in|K| \mid a \leq b_{L}(x) \leq 1\right\}$. Then $N_{a}(|L|)$ is a closed neighborhood of $|L|$ and if $x \in N(|L|)$ then $x=t y+(1-t) x^{\prime}$ where $x^{\prime} \in|L|$ is unique and $a \leq 1-t$. Define $r: N_{a}(|L|) \rightarrow|L|$ by $r_{a}(x)=x^{\prime}$ 
(where $x^{\prime}$ is as above). Then $r_{a}$ is a continuous retraction and $r_{a}^{-1}\left(x^{\prime}\right)$ is homeomorphic to the join of $x^{\prime}$ with the link complex

$$
L_{K}\left(x^{\prime}\right)=\left\{s \in K \mid s s^{\prime} \in K \text { where } s^{\prime} \text { is the open simplex of } K \text { containing } x^{\prime}\right\} \text {. }
$$

Theorem (5.7). If $H, \delta$ is a cohomology theory on $|K|$ which is locally constant on every open simplex and if $H, \delta$ is of finite type on $|K|$, then for every full subcomplex $L \subset K$ and every $0<a<1$ there is an isomorphism $\rho: H\left(N_{a}(|L|)\right) \approx H(|L|)$.

Proof. Let $H^{\prime}$ be the cohomology theory defined on $|L|$ by $H^{\prime}(A)=H\left(r^{-1} A\right)$ for closed $A \subset|L|$. There is a natural restriction homomorphism from $H^{\prime}$ to $H$ on $|L|$. By Corollary (5.6) $H^{\prime}\left(x^{\prime}\right) \approx H(x)$ for every $x \in|L|$. It follows that $H^{\prime}(|L|) \approx h(|L|)$ or $H\left(N_{a}(|L|) \approx H(|L|)\right.$.

Theorem (5.8). Let $H, \delta$ be a cohomology theory of finite type on a polyhedron $X=|K|$ and suppose $H$ is locally constant on every open simplex of $K$. Let $X_{i}=\left|K^{i}\right|$. Then there is a short exact sequence

$$
0 \rightarrow \lim ^{1}\left\{H^{q-1}\left(X_{i}\right)\right\} \rightarrow H^{q}(X) \rightarrow \underset{\lim }{\longleftarrow}\left\{H^{q}\left(X_{i}\right)\right\} \rightarrow 0 .
$$

Proof. Let $L$ be the barycentric subdivision of $K$ and let $L_{i}$ be the corresponding subdivision of $K^{i}$. Then each $L_{i}$ is a full subcomplex of $L$. Since $L_{i}$ is a subcomplex of $L_{i+1}$ it follows that $b_{L_{i}} \leq b_{L_{i+1}}(x)$ for every $x \in|L|$. Let $Y_{i}=N_{1-\left(1 / 2^{i}\right)}\left(\left|L_{i}\right|\right)$. Then $Y_{i} \subset$ int $Y_{i+1}$ and $\bigcup Y_{i}=X$. By Theorem (4.6) there is a short exact sequence

$$
0 \rightarrow \lim ^{1}\left\{H^{q-1}\left(Y_{i}\right)\right\} \rightarrow H^{q}(X) \rightarrow \underset{\lim }{\longleftarrow}\left\{H^{q}\left(Y_{i}\right)\right\} \rightarrow 0 .
$$

By Theorem (5.7), $\rho: H^{j}\left(Y_{i}\right) \approx H^{j}\left(\left|L_{i}\right|\right)=H^{j}\left(X_{i}\right)$ so that $\lim ^{1}\left\{H^{q-1}\left(Y_{i}\right)\right\} \approx$ $\lim ^{1}\left\{H^{q-1}\left(X_{i}\right)\right\}$ and $\lim \left\{H^{q}\left(Y_{i}\right)\right\} \approx \lim \left\{H^{q}\left(X_{i}\right)\right\}$. The result follows on substituting these isomorphic groups into the short exact sequence.

\section{A SPECTRAL SEQUENCE}

Throughout this section we assume $K$ is a simplicial complex and $H, \delta$ is an additive cohomology theory on $|K|$ which is locally constant on every open simplex $\langle s\rangle$ where $s \in K$. We shall show there is a stack of graded groups on $K$ and if $H$ is nonnegative or $K$ is finite dimensional there is a spectral sequence converging to the bigraded module associated to a filtration of $H(|K|)$ whose $E_{2}$-term is isomorphic to the cohomology of $K$ with coefficients in the stack. This specializes to give the Leray-Serre spectral sequence in cohomology of a simplicial map between simplicial complexes and to the Atiyah-Hirzebruch spectral sequence of $K$-theory for a finite dimensional simplicial complex. As a consequence of the existence of this spectral sequence we deduce the uniqueness theorem of Eilenberg-Steenrod for cohomology on simplicial complexes.

If $x, y \in\langle s\rangle$ where $s \in K$ there is an isomorphism $H(x) \approx H(y)$ corresponding to the local system $\mathscr{H}$ on $\langle s\rangle$ defined in (2.7) and this local system is a simple system because $\langle s\rangle$ is simply connected. We let $G^{q}(s)=G^{q}(x)$ for $x \in\langle s\rangle$, it being understood that $G^{q}(s)$ is independent of $x$ up to canonical isomorphism by the previous remark. If $s$ is a face of $s^{\prime}$ let $I$ be a copy of the unit interval embedded in $s^{\prime}$ so that $(0,1] \subset\left\langle s^{\prime}\right\rangle$ and $0 \in\langle s\rangle$. Since $H$ is locally constant on $(0,1]$ it follows from Theorem (3.5) that $\rho: H(I) \rightarrow H(\{0\})$ 
is an isomorphism. Then the composite $H(\{0\}) \stackrel{\rho^{-1}}{\rightarrow} H(I) \stackrel{\rho^{\prime}}{\rightarrow} H(\{1\})$ is a homomorphism $h_{s}^{s^{\prime}}: G^{q}(s) \rightarrow G^{q}\left(s^{\prime}\right)$ which is independent, up to canonical isomorphism, of the choice of $I$. If $s$ is a face of $s^{\prime}$ and $s^{\prime}$ is a face of $s^{\prime \prime}$, it is easily verified that $h_{s}^{s^{\prime \prime}}: G^{q}(s) \rightarrow G^{q}\left(s^{\prime \prime}\right)$ equals the composite

$$
G^{q}(s) \stackrel{h_{s}^{s^{\prime}}}{\rightarrow} G^{q}\left(s^{\prime}\right) \stackrel{h_{s^{\prime}}^{s^{\prime \prime}}}{\rightarrow} G^{q}\left(s^{\prime \prime}\right) .
$$

Thus $\mathscr{G}^{q}=\left\{G^{q}(s)\right\}_{s \in K}$ is a stack of coefficients on $K$ and we can form the $p$ th cohomology group $H^{p}\left(K ; \mathscr{G}^{q}\right)$ of $K$ with coefficients in this stack.

Let $A$ be a nonempty compact subset of $|K|$ contained in an open simplex $\langle s\rangle$. Then there is a set $B$ homeomorphic to a cube $I^{k}$ for some $k$ with $A \subset B \subset\langle s\rangle$. It follows from Corollary (3.3) that if $x \in A$ then $H(B) \rightarrow H(x)$ is an isomorphism. Therefore, $\rho: H(B) \rightarrow H(A)$ is a monomorphism and the reduced group $\widetilde{H}(A)$ is defined by $\widetilde{H}(A)=\operatorname{coker}[\rho: H(B) \rightarrow H(A)]$. This is well defined, for if $B_{1}, B_{2}$ are two subsets of $\langle s\rangle$ each containing $A$ and homeomorphic to a cube, there is a third set $B_{3} \subset\langle s\rangle$ containing $B_{1} \cup B_{2}$ and homeomorphic to a cube. If follows that $H\left(B_{3}\right) \rightarrow H\left(B_{1}\right)$ and $H\left(B_{3}\right) \rightarrow H\left(B_{2}\right)$ are isomorphisms so that

$$
\begin{aligned}
\operatorname{coker}\left[\rho_{1}: H\left(B_{1}\right) \rightarrow H(A)\right] & =\operatorname{coker}\left[\rho_{3}: H\left(B_{3}\right) \rightarrow H(A)\right] \\
& =\operatorname{coker}\left[\rho_{2}: H\left(B_{2}\right) \rightarrow H(A)\right] .
\end{aligned}
$$

Remarks. (6.1) $H(A) \approx H(B) \oplus \widetilde{H}(A) \approx H(x) \oplus \widetilde{H}(A)$ for $x \in A$ (where $A \subset B$ and $B$ is homeomorphic to a cube).

(6.2) If $A$ is homeomorphic to a cube, $\widetilde{H}(A)=0$.

Lemma (6.3). If $A, B$ are compact subsets in $\langle s\rangle$ with $A \cap B \neq 0$ there is an exact reduced $M V$ sequence

$$
\cdots \stackrel{\tilde{\delta}}{\rightarrow} \widetilde{H}^{q}(A \cup B) \stackrel{\tilde{\alpha}}{\rightarrow} \widetilde{H}^{q}(A) \oplus \widetilde{H}^{q}(B) \stackrel{\tilde{\beta}}{\rightarrow} \widetilde{H}^{q}(A \cap B) \stackrel{\tilde{\delta}}{\rightarrow} \widetilde{H}^{q+1}(A \cup B) \stackrel{\tilde{\alpha}}{\rightarrow} \cdots
$$

Proof. Let $C$ be a cube in $\langle s\rangle$ containing $A \cup B$. Then the exact MV sequence of $C, C$

$$
\cdots \rightarrow H^{q}(C) \stackrel{\alpha}{\rightarrow} H^{q}(C) \oplus H^{q}(C) \stackrel{\beta}{\rightarrow} H^{q}(C) \stackrel{\delta}{\rightarrow} H^{q+1}(C) \rightarrow \cdots
$$

is mapped by restriction monomorphically into the exact $\mathrm{MV}$ sequence of $A, B$

$$
\cdots \rightarrow H^{q}(A \cup B) \stackrel{\alpha}{\rightarrow} H^{q}(A) \oplus H^{q}(B) \stackrel{\beta}{\rightarrow} H^{q}(A \cap B) \stackrel{\delta}{\rightarrow} H^{q+1}(A \cup B) \rightarrow \cdots
$$

so the quotient sequence, which is the reduced MV sequence, is also exact.

Lemma (6.4). If $S^{p} \subset\langle s\rangle$, then $\widetilde{H}^{q}\left(S^{p}\right) \approx H^{q-p}(x)$ for $x \in\langle s\rangle$.

Proof. Represent $S^{p}$ as an increasing sequence of spheres

$$
S^{0} \subset S^{1} \subset \cdots \subset S^{p}
$$

such that for $0 \leq j \leq p, S^{j}$ divides $S^{j+1}$ into two closed hemispheres $E_{+}$, $E_{-}$such that $S^{j}=E_{+} \cap E_{-}$and $S^{j+1}=E_{+} \cup E_{-}$. By Lemma (6.3) there is an exact sequence

$$
\widetilde{H}^{q}\left(E_{+}\right) \oplus \widetilde{H}^{q}\left(E_{-}\right) \rightarrow \widetilde{H}^{q}\left(S^{j}\right) \stackrel{\tilde{\delta}}{\rightarrow} \widetilde{H}^{q+1}\left(S^{j+1}\right) \rightarrow \widetilde{H}^{q+1}\left(E_{+}\right) \oplus \widetilde{H}^{q+1}\left(E_{-}\right) .
$$


By Remark (6.2) the groups on both ends are zero so there are isomorphisms

$$
\widetilde{H}^{q-p}\left(S^{0}\right) \stackrel{\tilde{\delta}}{\approx} \widetilde{H}^{q-p+1}\left(S^{1}\right) \approx \cdots \stackrel{\tilde{\delta}}{\approx} \widetilde{H}^{q}\left(S^{p}\right) .
$$

Now $S^{0}=\left\{y_{1}, y_{2}\right\}$ and $\widetilde{H}^{q-p}\left(S^{0}\right) \approx\left[H^{q-p}(x) \oplus H^{q-p}(x)\right] /\{(a, b) \mid a, b \in$ $\left.H^{q-p}(x), a=b\right\}$. Therefore, the map sending $a \in H^{q-p}(x)$ to $(\overline{a, 0}) \in$ $\widetilde{H}^{q-p}\left(S^{0}\right)$ (where $(\overline{a, 0})$ denotes the image of $(a, 0) \in H^{q-p}\left(S^{0}\right)$ ) in $\widetilde{H}^{q-p}\left(S^{0}\right)$ ) is an isomorphism and $(\overline{0, a})=-(\overline{a, 0})$. Therefore, $H^{q-p}(x) \approx \widetilde{H}^{q}\left(S^{p}\right)$.

Corollary (6.5). If $S^{p} \subset\langle s\rangle$, then $H^{q}\left(S^{p}\right) \approx H^{q}(x) \oplus H^{q-p}(x)$ for $x \in\langle s\rangle$. Proof. This is immediate from Remark (6.1) and Lemma (6.4).

Lemma (6.6). Let $S^{j}=E_{+} \cap E_{-}, S^{j+1}=E_{+} \cup E_{-}$and $\tilde{\delta}: \widetilde{H}^{q}\left(S^{j}\right) \approx \widetilde{H}^{q+1}\left(S^{j+1}\right)$ be obtained from the reduced $M V$ sequence of $E_{+}, E_{-}$and $\tilde{\delta}^{\prime}: \widetilde{H}^{q}\left(S^{j}\right) \approx$ $\widetilde{H}^{q+1}\left(S^{j+1}\right)$ be obtained from the reduced $M V$ sequence of $E_{-}, E_{+}$(in the other order). Then $\tilde{\delta}^{\prime}=-\tilde{\delta}$.

Proof. Let $S_{1}^{j}$ be a copy of $S^{j}$ obtained by slightly rotating $S^{j+1}$ about an $S^{j-1}$ contained in $S^{j}$ and let $E_{+1}, E_{-1}$ be the images of $E_{+}, E_{-}$under this rotation. Let $B$ be the part of $S^{j+1}$ between $S^{j}$ and $S_{+}^{j}$ through which the rotation moves $S^{j}$ and let $F_{+}=E_{+} \cup E_{+1}, F_{-}=E_{-} \cup E_{-1}$. Then $B=F_{+} \cap F_{-}$ and $S^{j+1}=F_{+} \cup F_{-}$. Furthermore, each of $F_{+}, F_{-}$is a cube so $\widetilde{H}\left(F_{+}\right)=0=$ $\widetilde{H}\left(F_{-}\right)$, and it is easy to see, using methods similar to those in the proof of Corollary (3.2), that $H(B) \rightarrow H\left(S^{j}\right)$ and $H(B) \rightarrow H\left(S_{1}^{j}\right)$ are isomorphisms. By naturality of $\delta$, hence of $\tilde{\delta}$, there is a commutative diagram

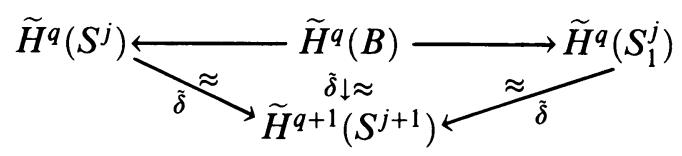

Thus, the composite across the top is an isomorphism $\widetilde{H}^{q}\left(S^{j}\right) \approx \widetilde{H}^{q}\left(S_{1}^{j}\right)$ commuting with the map $\tilde{\delta}$.

This isomorphism corresponds to a small rotation of $S^{j+1}$ taking $S^{j}$ into $S_{1}^{j}$. Consider a rotation of $S^{j+1}$ taking $S^{j}$ into $S^{j}$ but interchanging the two hemispheres of $S^{j}$ and of $S^{j+1}$. This rotation can be accomplished by a sequence of small rotations for each of which there is the isomorphism above. Composing these gives an isomorphism $\lambda: \widetilde{H}^{q}\left(S^{j}\right) \approx \widetilde{H}^{q}\left(S^{j}\right)$ corresponding to interchanging the two hemispheres of $S^{j-1}$ in $S^{j}$ and yielding a commutative diagram

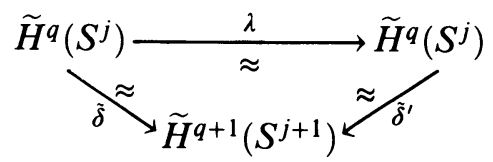

We prove the lemma by induction on $j$. If $j=0$, there is a commutative diagram

$$
\widetilde{H}^{q}(\{x, y\}) \underset{\tilde{\delta}}{\approx} \underset{\widetilde{H}^{q+1}\left(S^{1}\right)^{2}}{\approx} \underset{\tilde{H}^{q}(\{x, y\})}{\approx}
$$


where the map $\lambda$ corresponds to interchanging $x$ and $y$. Thus, $\lambda(\overline{g, 0})=$ $(\overline{0, g})=-(\overline{g, 0})$ and so $\tilde{\delta}^{\prime}(-(\overline{g, 0}))=\tilde{\delta}(\overline{g, 0})$ or $\tilde{\delta}^{\prime}=-\tilde{\delta}$ in this case.

Assume $j>0$ and the result is true in dimension $j-1$. We have a commutative diagram

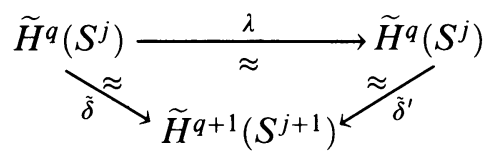

where the isomorphism $\lambda$ commutes in the diagram

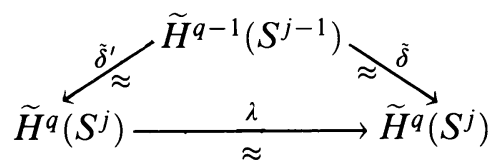

By the inductive hypothesis (since $\tilde{\delta}$ is an isomorphism), $\lambda(a)=-a$ for $a \in$ $\widetilde{H}^{q}\left(S^{j}\right)$. Therefore, $\tilde{\delta}^{\prime}(-a)=\tilde{\delta}(a)$ for $a \in \widetilde{H}^{q}\left(S^{j}\right)$ so $\tilde{\delta}^{\prime}=-\tilde{\delta}$ in $S^{j}$.

Let $A^{0}, \ldots, A^{p}$ be vertices of a simplex $t$ in $\langle s\rangle$ given in some linear order. For $g \in H^{q-p+1}(x), x \in\langle s\rangle$ we associate an element $g A^{0} \cdots A^{p} \in \widetilde{H}^{q}\left({ }^{\circ}\right)$ such that

(1) $g A^{0} A^{1}=(\overline{0, g}) \in \widetilde{H}^{q}(\dot{t})$ if $p=1$,

(2) $g A^{0} \cdots A^{p}=\tilde{\delta}\left(g A^{1} \cdots A^{p}\right)$ if $p>1$ where if $t^{\prime}=\left|A^{1} \cdots A^{p}\right|$ then $\tilde{\delta}: H^{q-1}\left(t^{\prime}\right) \underset{\approx}{\approx} H^{q}(t)$ is from the reduced $M V$ sequence of $\left|A^{0} t^{\prime}\right|,\left|t^{\prime}\right|$.

Theorem (6.7). If $\omega$ is a permutation of $0, \ldots, p$ then $g A^{\omega(0)} \cdots A^{\omega(p)}=$ $(\operatorname{sgn} \omega) A^{0} \cdots A^{p}$.

Proof. It suffices to verify this for a transposition. We use induction on $p$. If $p=1, g A^{1} A^{0}=(\overline{0, g}) \in H^{q}\left(A^{1}\right) \oplus H^{q}\left(A^{0}\right)$ so $g A^{1} A^{0}=(\overline{g, 0}) \in H^{q}\left(A^{0}\right) \oplus$ $H^{q}\left(A^{1}\right)$. Since $0=(\overline{g, g})=(\overline{g, 0})+(\overline{0, g})$ we see that $g A^{1} A^{0}=-(\overline{0, g}) \in$ $H^{q}\left(A^{0}\right) \oplus H^{q}\left(A^{1}\right)$ and $g A^{1} A^{0}=-g A^{0} A^{1}$.

Assume $p>1$ and the result is valid for permutations of a $(p-1)$-simplex. If $\omega$ is a transposition of $A^{0}, \ldots, A^{p}$ leaving $A^{0}$ fixed then

$$
\begin{aligned}
g A^{\omega(0)} A^{\omega(1)} \cdots A^{\omega(p)} & =g A^{0} A^{\omega(1)} \cdots A^{\omega(p)}=\tilde{\delta}\left(g A^{\omega(1)} \cdots A^{\omega(p)}\right) \\
& =\tilde{\delta}\left(-g A^{1} \cdots A^{p}\right)=-g A^{0} \cdots A^{p}
\end{aligned}
$$

so the result is valid in this case. It remains to consider only the transposition $\omega(0)=1$ and $\omega(1)=0$. It follows from Lemma 6.6 that $g A^{0} A^{1} \cdots A^{p}=$ $-g A^{1} A^{0} A^{2} \cdots A^{p}$ so the result is valid in all cases and the theorem is proved.

Theorem (6.8). Let $A^{0}, \ldots, A^{p}$ be vertices of a simplex in $\langle s\rangle$ where $s$ is a face of $s^{\prime}$ and let $A^{0^{\prime}}, \ldots, A^{p^{\prime}}$ be vertices of a simplex in $\left\langle s^{\prime}\right\rangle$ with an embedding of $\left(A^{0} \cdots A^{p}\right) \times I$ in $\left|s^{\prime}\right|$ so that $\left|A^{0} \cdots A^{p}\right| \times(0,1] \subset\left\langle s^{\prime}\right\rangle,\left(A^{i}, 0\right)$ goes to $A^{i}$ and $\left(A^{i}, 1\right)$ to $A^{i^{\prime}}$. If $h_{s}^{s^{\prime}}: G^{q}(s) \rightarrow G^{q}\left(s^{\prime}\right)$ is the homomorphism of the stack $\mathscr{G}^{q}$, then $h_{s}^{s^{\prime}}(g) A^{0^{\prime}} \cdots A^{p^{\prime}}$ corresponds to $g A^{0} \cdots A^{p}$ under the composite

$$
\widetilde{H}^{q}\left(\left|A^{0} \cdots A^{p}\right|\right) \underset{\approx}{\leftarrow} \widetilde{H}^{q}\left(\left|A^{0^{\prime}} \cdots A^{p}\right| \times I \stackrel{\rho}{\rightarrow} \widetilde{H}^{q}\left(\left|A^{0^{\prime}} \cdots A^{p^{\prime}}\right|\right)\right.
$$

Proof. This follows by straightforward induction on $p$. 
Theorem (6.9). If $K$ is finite dimensional or $H, \delta$ is nonnegative there is a spectral sequence converging to the bigraded module associated to the filtration of $H^{n}(|K|)$ given by $F^{p} H^{n}(|K|)=\operatorname{ker}\left[H^{n}(|K|) \rightarrow H^{n}\left(\left|K^{p}\right|\right)\right]$ and with $E_{2}$-term such that $E_{2}^{p, q} \approx H^{p}\left(K ; \mathscr{G}^{q}\right)$.

Proof. As in Theorem (5.7) there is a closed neighborhood $N$ of $\left|K^{p-1}\right|$ in $\left|K^{p}\right|$ with $H(N) \approx H\left(\left|K^{p-1}\right|\right)$ and such that for each cell $s_{j}^{p} \in K^{p}-K^{p-1}$, $\left|s_{j}^{p}\right|-N$ is an open $p$-simplex $\left\langle t_{j}^{p}\right\rangle$ whose closure $t_{j}^{p} \subset\left\langle s_{j}^{p}\right\rangle$. Then $\left|K^{p}\right|=$ $N \cup \bigcup_{j} t_{j}^{p}$ and $N \cap \bigcup_{j} t_{j}^{p}=\bigcup i_{j}^{p}$. Since $H, \delta$ is additive, $H^{n}\left(\bigcup_{j} t_{j}^{p}\right) \stackrel{\sigma}{\approx} \prod_{i} H^{n}\left(t_{j}^{p}\right)$ and $H^{n}\left(\bigcup_{j} i_{j}^{p}\right) \stackrel{\sigma}{\approx} \prod_{j} H^{n}\left(\dot{t}_{j}^{p}\right)$. By exactness of the MV sequence of $\bigcup_{j} t_{j}^{p}, N$ we obtain the exact sequence

$$
\cdots \stackrel{\alpha}{\rightarrow} \prod_{j} H^{n}\left(\dot{t}_{j}^{p}\right) \oplus H^{n}(N) \stackrel{\rho_{j}}{\rightarrow} \prod_{j} H^{n}\left(\dot{t}_{j}^{p}\right) \stackrel{\delta}{\rightarrow} H^{n+1}\left(\left|K^{p}\right|\right) \stackrel{\alpha}{\rightarrow} \cdots .
$$

By definition of the reduced group, for each $j$, there is an exact sequence $0 \rightarrow H^{n}\left(t_{j}^{p}\right) \stackrel{\rho_{j}}{\rightarrow} H^{n}\left(\dot{t}_{j}^{p}\right) \rightarrow \widetilde{H}^{n}\left(\dot{t}_{j}^{p}\right) \rightarrow 0$. We also have a product exact sequence

$$
\cdots \rightarrow \prod_{j} H^{n}\left(\dot{t}_{j}^{p}\right) \underset{\approx}{\rightleftarrows} \operatorname{im} \rho_{j} \rightarrow 0 \rightarrow \prod_{j} H^{n+1}\left(\dot{t}_{j}^{p}\right) \rightarrow \cdots
$$

which is a subsequence of $(*)$. Therefore, the quotient sequence is an exact sequence and this is the sequence

$$
\cdots \rightarrow H^{n}(N) \rightarrow \prod_{j} \widetilde{H}^{n}\left(\dot{t}_{j}^{p}\right) \rightarrow H^{n+1}\left(\left|K^{p}\right|\right) \rightarrow H^{n+1}(N) \rightarrow \cdots .
$$

Because $H^{n}(N) \approx H^{n}\left(\left|K^{p-1}\right|\right)$, we have an exact sequence

$$
\cdots \rightarrow H^{n}\left(\left|K^{p-1}\right|\right) \rightarrow \prod_{j} \widetilde{H}^{n}\left(\dot{t}_{j}^{p}\right) \rightarrow H^{n+1}\left(\left|K^{p}\right|\right) \rightarrow H^{n+1}\left(\left|K^{p-1}\right|\right) \rightarrow \cdots .
$$

If $K$ is finite dimensional, certainly $H^{n}\left(\left|K^{p}\right|\right)=H^{n}\left(\left|K^{p-1}\right|\right)$ for $p$ large enough. If $H, \delta$ is nonnegative, then by Lemma (6.4),

$$
\widetilde{H}^{n}\left(\dot{t}_{j}^{p}\right) \approx H^{n-p+1}\left(x_{j}\right) \approx G^{n-p+1}\left(s_{j}^{p}\right)
$$

and this is 0 if $p>n+1$ so that, in this case, $H^{n}\left(\left|K^{p}\right|\right) \approx H^{n}\left(\left|K^{p-1}\right|\right)$ if $p$ is large enough. In either case it follows by Theorem (5.8) and Example (4.2) that $\lim ^{1}\left\{H^{n-1}\left(\left|K^{p}\right|\right)\right\}=0$ and $H^{n}(|K|) \approx \lim \left\{H^{n}\left(\left|K^{p}\right|\right)\right\}$. By using exact couples there is a spectral sequence converging to the bigraded group of the filtration on $H^{n}(|K|)$ given by

$$
F^{p} H^{n}(|K|)=\operatorname{ker}\left[H^{n}(|K|) \rightarrow H^{n}\left(\left|K^{p}\right|\right)\right]
$$

and with $E_{1}^{p, q} \approx \prod_{j} \widetilde{H}^{p+q-1}\left(i_{j}^{p}\right)$. Now $t_{j}^{p}$ is a simplex in $\left\langle s_{j}^{p}\right\rangle$ whose vertices $B^{0}, \ldots, B^{p}$ correspond to the vertices $A^{0}, \ldots, A^{p}$ of $s_{j}^{p}$. Thus, for each order $A^{0}, A^{1}, \ldots, A^{p}$ of the vertices of $s_{j}^{p}$ there is a corresponding order $B^{0}, B^{1}, \ldots, B^{p}$ of the vertices of $t_{j}^{p}$ and every element of $\widetilde{H}^{n}\left(\dot{t}_{j}^{p}\right)$ has the form $g B^{0} \cdots B^{g}$ for some $g \in G^{n-p+1}\left(s_{j}^{p}\right)$. Therefore, by Theorem (6.7), an element of $\prod_{j} \widetilde{H}^{n}\left(\hat{t}_{j}^{p}\right)$ corresponds to a function $c$ from orderings of the vertices $A^{0}, A^{1}, \ldots, A^{p}$ of the simplex $s_{j}^{p} \in K^{p}-K^{p-1}$ to 
$G^{n-p+1}\left(s_{j}^{p}\right)$ for each $j$ which is alternating (i.e. for any permutation $\omega$ of $\left.\{0,1, \ldots, p\}, c\left(A^{\omega(0)} \cdots A^{\omega(p)}\right)=(\operatorname{sgn} \omega) c\left(A^{0} \cdots A^{p}\right)\right)$. Thus, $c$ is a cochain of $C^{p}\left(K ; \mathscr{G}^{n-p+1}\right)$. It follows from Theorem (6.8) that the differential $d_{1}$ of the $E_{1}$-term corresponds to the coboundary operator of the complex $C^{*}\left(K ; \mathscr{G}^{n-p+1}\right)$ so that the $E_{2}$-term has $E_{2}^{p, q} \approx H^{p}\left(K ; \mathscr{G}^{q}\right)$.

Corollary (6.10). Let $H, \delta$ and $H^{\prime}, \delta^{\prime}$ be two additive cohomology theories on $|K|$ such that $|K|$ is extensive for both and suppose that $H^{q}(x)=0=H^{\prime q}(x)$ for $q \neq 0$ and $H^{0}(x) \approx H^{\prime 0}(x)$ for some $x \in|K|$. Then $H^{n}(|K|) \approx H^{\prime n}(|K|)$ for all $n$.

Proof. The assumption on $H$ implies that in the spectral sequence of Theorem (6.9) the only nonzero terms in $E_{2}$ are $E_{2}^{p, 0}$ so that $E_{\infty}^{p, 0} \approx E_{2}^{p, 0}$ is the only nonzero term of $E_{2}$ and $H^{n}(|K|) \approx E_{\infty}^{n, 0} \approx E_{2}^{n, 0} \approx H^{n}\left(K ; \mathscr{G}^{0}\right)$. Similarly, $H^{\prime n}(|K|) \approx H^{n}\left(K ; \mathscr{G}^{\prime 0}\right)$. The hypothesis that $|K|$ is extensive for both $H$ and $H^{\prime}$ implies that $\mathscr{G}^{0}$ and $\mathscr{G}^{\prime 0}$ are simple systems and since $H^{0}(x) \approx H^{\prime 0}(x)$ for some $x$ it follows that $\mathscr{G}^{0} \approx \mathscr{G}^{\prime 0}$. Therefore, $H^{n}(|K|) \approx H^{\prime n}(|K|)$.

In case $K$ is a finite complex the hypothesis of additivity is not needed in Corollary (6.10) and so we obtain the uniqueness theorem of EilenbergSteenrod as a special case of Corollary (6.10).

\section{REFERENCES}

1. S. Eilenberg and N. E. Steenrod, Foundations of algebraic topology, Princeton Univ. Press, Princeton, N. J., 1952.

2. J. W. Keesee, On the homotopy axiom, Ann. of Math. (2) 54 (1951), 247-249.

3. J. Milnor, On axiomatic homology theory, Pacific J. Math. 12 (1962), 337-341.

4. W. S. Massey, Homology and cohomology theory, Dekker, New York, 1978.

5. E. Spanier, Cohomology isomorphisms, Contemp. Math. 12 (1982), 315-329.

6. __ Cohomology with supports, Pacific J. Math. 123 (1986), 447-464.

7. __ Cohomology theories on spaces, Trans. Amer. Math. Soc. 301 (1987), 149-161.

8. __, Weakly additive cohomology, Publ. Math. 34 (1990), 145-150.

Department of Mathematics, University of California, Berkeley, California 94720 